\title{
14. PULSARS AND X-RAY SOURCES
}

\author{
F. PACINI \\ Laboratorio di Astrofisica Spaziale, Frascati, Italy
}

\begin{abstract}
We summarize some theoretical aspects of the X-ray emission from genuine pulsars and their relation to the more general problems of $\mathrm{X}$-ray sources.
\end{abstract}

Most galactic X-ray sources appear to be connected with the final stages of stellar evolution. This is evident for the sources identified with SN remnants; however, even in the case of objects like Sco X-1 or Cen X-3, spectral information and/or the presence of fast periodicities clearly imply that one is dealing with condensed stars such as white dwarfs, neutron stars or, perhaps, black holes.

When discussing the basic nature of these objects the question naturally arises of a possible link between X-ray sources and the pulsar mechanism. The possibility of this connection has been discussed before the discovery of periodic X-ray sources but interest in the subject has been greatly enhanced by the recent observational results.

In the following we comment briefly about some aspects of this relationship.

\section{X-Ray Emission from Genuine Pulsars}

Only one genuine pulsar, NP 0532, has been detected as an X-ray source. This indicates that the X-ray output of pulsars dies out very rapidly when the star slows down. This observational evidence can be understood if the optical and the X-ray luminosity of pulsars arise through the synchrotron emission by particles moving in the proximity of the speed of light distance. It has been shown (Pacini, 1971) that in this case the synchrotron output evolves roughly like $M^{4} \cdot P^{-10}(M$ is the magnetic moment of the star and $P$ the rotation period). This accounts for the lack of optical and X-ray emission from most pulsars.

It cannot be ruled out, however, that some genuine very fast pulsars exist among the known X-ray souces. The lack of a SN remnant around these sources would not pose any particular problem if their age considerably exceeds $10^{4} \mathrm{yr}$. The existence of pulsars born with 'weak magnetic fields' $\left(\$ 10^{11} \mathrm{G}\right)$ would naturally lead to the existence of fast, old pulsars. This could form a population of compact non-thermal $\mathrm{X}$-ray sources similar to NP 0532 and with a typical output $\simeq 10^{36}-10^{37} \mathrm{erg} \mathrm{s}^{-1}$. The space distribution of these sources would resemble that of normal radio pulsars.

Genuine pulsars with very long periods $\gtrsim 1 \mathrm{~s}$ could only arise around a rotating, magnetic white dwarf (the larger value of $M$ could compensate for the long period!) 


\section{Pulsars as Motors for X-Ray Sources}

Genuine pulsars produce relativistic particles and can provide the energy source for the X-ray emission from SN remnants. This is the well known case of the pulsar NP 0532 in the Crab Nebula. It is not yet clear whether the X-ray emission from some other SN remnants has a non-thermal origin: if so, this would require the existence of a central (as yet undiscovered) pulsar.

Most of theoretical discussion of pulsar electrodynamics is centered upon the unipolar inductor theory (Goldreich and Julian, 1969) or the magnetic dipole radiation theory (Pacini, 1967, 1968; Ostriker and Gunn, 1969). The great success of these theories is due to the fact that they explain the overall energetics of the pulsar and also provide a simple and attractive scheme for investigating pulsar electrodynamics.

In particular both theories account for the production of very fast particles. However, there is a deep disagreement between the observed energy spectrum of the particles injected into the Crab Nebula (a power law between roughly $10^{8} \mathrm{eV}$ and $10^{14} \mathrm{eV}$ ) and the monoenergetic character of the spectrum predicted by the simple versions of the above mentioned theories. There is little doubt that future investigation of the acceleration should abandon the 'test particle' approach and rather deal with the more complicated problem of the mutual interaction of plasmas and rotating electromagnetic fields. (Both in the near zone and in the far-wave region).

Finally, we recall (Davidson et al., 1971) that sources like Sco X-1 could perhaps be energized by a central pulsar hidden within a cocoon. If this is the case, and if the motor for thermal sources is indeed of a highly non-thermal nature, the heating of cocoon could be accompanied by a conspicuous flux of non-thermal $\gamma$-rays produced by bremsstrahlung or by nuclear processes. The detection of $\gamma$-rays from thermal $\mathrm{X}$-ray sources would represent an important indication in favour of the existence of a central non-thermal motor.

\section{Periodic X-Ray Sources}

The recent discovery of the periodic sources Cen X-3 and Hercules has attracted a great interest. Possible explanations are discussed elsewhere in this volume. It appears clear that these sources have little to do with genuine pulsars like NP 0532. Their mechanism seems instead intimately related to the evolution of a close binary system when one of the two stars reaches the end point of stellar evolution and accretes mass from the companion.

\section{Acknowledgements}

I am especially grateful to Drs. A. Cavaliere and E. E. Salpeter for a very stimulating collaboration and for interesting discussions. This work has been partly supported by NATO Grant 601. 


\section{References}

Davidson, K., Pacini, F., and Salpeter, E. E.: 1971, Astrophys. J. 168, 45.

Goldreich, P. and Julian, W.: 1969, Astrophys. J. $157,869$.

Ostriker, J. and Gunn, J.: 1969, Astrophys. J. 157, 1395.

Pacini, F.: 1967, Nature 216, 567.

Pacini, F.: 1968, Nature 219, 145.

Pacini, F.: 1971, Astrophys. J. Letters 163, L17. 